\title{
Wir sind für Sie nah - Ebewe
}

Die Firma Ebewe macht es sich zur Aufgabe, eine ganzheitliche Krebstherapie zu verwirklichen - Worte in Taten umzusetzen!

Mit der breitesten Palette an Medikamenten in der Chemo- und Hormontherapie zählen wir zu den Spezialisten in der Onkologie.

Als österreichisches Unternehmen hilft uns unser Produktions- und Forschungsstandort in Unterach am Attersee, die Wege kurz zu halten, schnell und individuell zu handeln.

Neben der räumlichen Nähe gibt es noch einen zweiten wesentlichen Faktor den der menschlichen Nähe.

Diese hat für die Ebewe-Mitarbeiter eine besondere Bedeutung, denn in der Kette zwischen Vorsorge, Diagnostik, Therapie und Nachsorge geht oft der Mensch als Mensch verloren. Die Patienten brauchen aber Kraft für ihre Therapie, Mut, um wieder neu anzufangen, und einfühlsame Partner, um mit ihnen über ihre Krankheit zu sprechen.

Inzwischen wächst die Bereitschaft aller, die am Therapiegeschehen beteiligt sind, sich mit der seelischen Not der Patienten auseinanderzusetzen und nach Hilfsmöglichkeiten zu suchen. Im Interesse der Betroffenen unterstützen wir diese Bemühungen, z.B. mit unserem psychoonkologischen Seminarkonzept für Ärzte.

Gemeinsam nehmen wir die Herausforderung «Krebs» an:

Apotheker + Pflegepersonal + Patient + Arzt + Angehörige + Ebewe . 